\title{
UN ANÁLISIS DE LAS PATOLOGÍAS MUSCULOESQUELÉTICAS EN CLARINETISTAS
}

\author{
Juan Granda Vera, Ma Loreto Lledó Sempere y José C. Barbero Álvarez
}

Grupo de Investigación "Análisis del Movimiento Humano" - Universidad de Granada jgranda@ugr.es-malolle@hotmail.com-jcba@ugr.es

\begin{abstract}
Existen pocos estudios sobre las patologías que padecen instrumentistas musicales, y aún son más escasos aquellos que se dedican plenamente al estudio de clarinetistas. En el presente estudio han participado un total de 38 clarinetistas, utilizándose un cuestionario diseñado por los autores de este trabajo a partir de los utilizados en estudios previos. Los datos encontrados revelan como patologías más relevantes el dolor de espalda $(26,3 \%)$ y la tendinitis en la mano derecha $(21,1 \%)$, detectándose como posibles causas una mala postura corporal en el momento de tocar (34\%) y un aumento en el tiempo de estudio $(26,4 \%)$.
\end{abstract}

There are few studies about pathologies suffered by musical instrumentalists, and less dedicated to clarinet players. This article reports a study with a total of 38 clarinet players, who answered a questionnaire designed by the authors based on questionnaires used in previous studies. The data show that the most important pathologies were backache $(26,3 \%)$ and tendinitis in right hand $(21,1 \%)$, detecting as possible causes a bad posture when playing (34\%) and an increase in the practising time (26,4\%).

\section{INTRODUCCIÓN}

En la acción de tocar el clarinete participan la gran mayoría de los músculos de nuestro cuerpo, desde las piernas hasta la cara, pero existen algunos músculos que actúan más activamente en el momento de la ejecución del instrumento, ayudando a que la posición de la colocación del cuerpo sea la más correcta posible como es el caso de las extremidades superiores (CANTó, 2008), ya que de ello depende la sujeción del clarinete y la posición correcta de los dedos en el momento de tocar. De gran importancia es la conexión entre los brazos y otras partes del cuerpo como el tórax y la espalda, para una correcta colocación en el momento de tocar el clarinete.

De entre los grupos musculares que participan en la interpretación instrumental de los clarinetistas vamos a definir aquellos más relevantes por su potencial relación con las patologías que en ellos pudieran generarse. Respecto a los músculos de la parte superior del tronco, cabe destacar el trapecio, situado en la región posterior del cuello y del tronco músculo que se divide en tres secciones de fibras, siendo las fibras superiores las encargadas de la elevación del hombro y la aproximación del omóplato a la columna vertebral, mientras que las fibras intermedias están interconectadas con los abductores del brazo y las fibras inferiores son las responsables de la rotación del omóplato.

Para los clarinetistas es importante que toda la zona del trapecio esté lo más relajada posible (sobre todo las fibras superiores) ya que, por la posición corporal que se adopta con el instrumento, se tiende a elevar los hombros produciendo tensión en toda la zona superior de la espalda y por lo tanto una mala costumbre postural. Debido a esto, son muchas las ocasiones en las que aparecen molestias y la persistencia de esta posición puede desarrollar una contracción sostenida e involuntaria de algunas fibras o fascículos musculares en la zona (comúnmente denominada contractura muscular), debido a posturas no anatómicas repetitivas y forzadas y/o sobrecarga muscular continuada provocando que la ejecución del instrumento sea más dolorosa y molesta.

El músculo angular del omóplato tiene varias funciones, aunque las más importantes en el momento de tocar son la elevación y aproximación del omóplato a la columna vertebral, contribuyendo con ello a un descenso del hombro, y la inclinación de la cabeza (columna cervical) hacia el lado correspondiente. Cuando se toca el clarinete, la primera de sus funciones es la de ayudar a la correcta colocación de los brazos, con la aproximación del omóplato, ya que todos los músculos que actúan para realizar esta acción están interconectados entre sí. La segunda de estas funciones (la inclinación de la cabeza-columna cervical) actúa en el momento de tocar, adaptando sobre todo la posición correcta de la cabeza, ya que ayuda a que ésta se mantenga recta en todo 
momento, intentando que no se produzca una elevación de los hombros. El deltoides anterior o porción clavicular tiene como función la rotación interna y la flexión del hombro, teniendo que estar en equilibrio con el deltoides posterior o porción espinal para una correcta colocación, y entra en acción cuando nos disponemos a colocar hacia delante los brazos para la sujeción del clarinete. Por último, el bíceps es el responsable de la rotación del antebrazo y la flexión del codo. Actúa en menor medida sobre el hombro y en conjunto con los músculos supinador largo y braquial anterior. En los clarinetistas ayuda a flexionar correctamente los codos para poder sostener el instrumento.

En lo que respecta a la musculatura de la mano y de la muñeca, el músculo flexor común de los dedos tiene como función la flexión de los dedos y su función en el momento de tocar el clarinete es pulsar las diferentes llaves y abrir y tapar los agujeros es preparar el instrumento para producir el sonido deseado. Los músculos flexor corto y flexor largo del pulgar se encargan de la flexión y la aproximación del pulgar hacia el resto de los dedos y, en la mano derecha, soportan todo el peso del clarinete, tratándose de los músculos de la mano que más problemas causan a los clarinetistas. El extensor común de los dedos se encuentra situado en la parte media de la superficie dorsal del antebrazo y su función es la extensión de los dedos meñique, anular, corazón e índice, y en el clarinetista entra en funcionamiento cada vez que los dedos meñique, anular, corazón e índice de ambas manos se levantan de las llaves o de los agujeros. Por último, los músculos lumbricales, que son músculos intrínsecos caracterizados por tener sus orígenes e inserciones en la misma mano, son los responsables de la flexión de la articulación metacarpofalángica de los dedos índice al meñique, así como la extensión simultánea de las falanges (HISLOP y MONTGOMERY, 2002). En el momento de tocar el clarinete ayuda a tener los dedos colocados correctamente, aproximándolos a los agujeros y a las llaves, para una correcta colocación de la mano en el momento en que se empiece a tocar.

\section{ESTUDIOS SOBRE LAS PATOLOGÍAS MUSCULOESQUELÉTICAS EN LOS INSTRUMENTISTAS MUSICALES}

La gran mayoría de problemas musculares que aparecen a lo largo de los años de estudio y profesionalización de los instrumentistas vienen producidos por malos hábitos posturales y de movimiento, adquiridos, muchos de ellos, desde los primeros años del estudio del instrumento (ROSSET y FÀBREGES, 2005), así como por una mala técnica de ejecución, haciéndose más evidentes estos problemas cuando ese estudio se incrementa en horas y en esfuerzo físico, produciendo molestias y dolores que impiden poder tocar con total normalidad y libertad (GREER y PANUSH, 1994; OROZCO y CERVELLO, 1996).

Las lesiones más importantes que padecen los instrumentistas se producen en la parte superior del tronco, los brazos y las manos (JOUBREL y sus colaboradores, 2001; CANTÓ, 2008; Elshensohn, 2010; SCHARF, 2010; DieTHELM, 2010). La aparición de estas dolencias puede ser motivadas por varias causas como la fisonomía del instrumento, la utilización de una técnica determinada, aumento del estudio, factores externos o incluso por la autoestima de cada instrumentista (PoTTER y JONES, 1995; ElSHENSOHN, 2010). Son bastantes los instrumentistas a los que les da miedo reconocer y aceptar que tienen algún tipo de dolencia física producida por tocar, ya que les produce vergüenza reconocerlo y hasta puede llegar a ser un riesgo laboral, por lo que ante este tipo de problemas, deciden disimularla, perjudicándose a sí mismos mucho más, como se indica en multitud de estudios publicados (ROSSET, 2003; GREER y PANUSH, 1994; PotTER y JONES, 1995; Hoppmann y PAtron, 1989; Joubrel, Robineau, Pétrilli y Gallien, 2001; Rosset, 2004; DOMMERHOLT, 2010; SCHAWARZENBACH, 2010).

ROSSET, RosinÉS y SALÓ (2000) abordaron la detección de los problemas físicos en músicos de Cataluña. Los participantes en el estudio fueron 1730 músicos catalanes, tanto estudiantes como profesionales, con al menos 2 años de experiencia con un instrumento musical. Este estudio no se centra únicamente en un instrumento, sino en todas las familias de instrumentos. Los datos reflejan 
que el 77,9\% de los encuestados han sufrido algún tipo de patologías en su vida musical. En este mismo estudio se indica que las patologías más comunes entre los encuestados han sido las musculoesqueléticas con un porcentaje del $85,7 \%$. Respecto a las causas de su aparición existe una amplia diversidad, presentando un mayor porcentaje el aumento de horas de ensayo $(43,8 \%)$, seguida por causas que el propio instrumentista desconocía $(24,55 \%)$ y por el aumento de trabajo $(22,6 \%)$. Son destacables los factores de riesgo como la edad en la que ocurre más a menudo la dolencia (entre los 31y 40 años), el curso de estudio (sobre todo a partir de $5^{\circ}$ grado medio de música), así como la dedicación y los cambios en la forma diaria de estudio producido por un examen o audición.

JOUBREL y sus colaboradores (2001) indagaron sobre las enfermedades musculoesqueléticas de los músicos. En dicho estudio participaron un total de 635 músicos de diferentes estilos musicales como música de cámara o jazz, sin especificar los instrumentos que interpretaban, de los cuales solo fueron válidos 141 cuestionarios. Del total de participantes, el 76,6\% habían sufrido una o varias patologías derivadas de la práctica musical, siendo las más comunes el síndrome del sobreuso muscular $(58,1 \%)$, el síndrome del túnel carpiano (17\%) y la distonía focal $(5,7 \%)$. En términos generales, las dolencias se localizaron mayoritariamente en la columna $(60,9 \%)$, sobre todo en la parte cervical y dorsal, y el dolor en las extremidades superiores $(52,5 \%)$, siendo la franja de edad en que más patologías se producían entre 30 y 35 años. Por género, las mujeres tendían a ser más propensas a dichas patologías, sobre todo al síndrome del sobreuso muscular.

Respecto a la distonía focal, ALTENMÜLLER (2003) señaló que el 1\% de los músicos estaba afectado por ella. JABUSCH y ALTENMÜLLER (2006) llevaron a cabo un estudio con 144 músicos profesionales (116 hombres y 28 mujeres) que la padecían, con un media de edad de 33 años (rango 17-63), en el Instituto de Fisiología de la Música de la Universidad de Hannover, siendo relevante la proporción en la ratio hombre/mujer $4: 1$. El porcentaje mayor de afectados eran instrumentistas de piano y guitarra, seguidos de instrumentistas de violín, flauta travesera y clarinete. Respecto a la edad, los datos promedio de los participantes en este estudio concordaban con los obtenidos en estudios similares sobre esta patología (BRANDFONBRENER y ROBSON, 2002; ALTENMÜLLER, 2003). En cuanto a los factores de carácter externo que parecían provocar su aparición, los autores señalaron las condiciones psicológicas (exceso de perfeccionismo y ansiedad). BARR y BARBE (2002) y COMELLA y sus colaboradores (2003) añadían como factores de carácter externo la práctica excesiva, la utilización de una nueva técnica y los movimientos repetitivos de forma estresante.

FRANK y VON MÜHLEN (2007) analizaron los problemas musculoesqueléticos de los músicos. Los datos ponen de manifiesto que el $70 \%$ de los músicos de las orquestas sufre algún tipo de dolencia derivado por su trabajo. Respecto a los factores de riesgo, incluye una anotación no realizada en el estudio anterior, el tamaño del instrumento, ya que las posiciones corporales que se adoptan en ocasiones son poco ergonómicas. Como posibles patologías encontramos en dicho estudio el sobreuso muscular, tendinitis, tendinitis de Quervein, afectando en su mayoría en las manos y en los miembros superiores. Una de las causas de estas patologías es una técnica deficiente, unida al esfuerzo físico que se produce y el trabajo que se realiza. Otras de las causas que señala es el repertorio, ya que en ocasiones no son los más correctos y su dificultad puede producir o acentuar dichas dolencias. Factores ligados a la forma de estudiar también pueden afectar a la posible aparición de patologías, mucho tiempo de estudio continuado, falta de descanso. Los datos también indican que las mujeres son más propensas a padecer patologías (entre un $67 \%$ y un $76 \%$ ). Los hombres sufren estas patologías entre un 52\% y un $63 \%$, siendo las posibles causas de ello un menor nivel de desarrollo muscular de las mujeres, así como las manos más pequeñas respecto a los hombres.

En un estudio más reciente, DOMMERHOLT (2010) estudia también sobre las patologías de músicos de diferentes modalidades instrumentales, sin especificar tipos de instrumentistas examinados sino abordando el examen de los problemas físicos de los músicos con carácter general, analizando diferentes aspectos de la interpretación del músico. Una de las cuestiones que aborda es la referida a la postura del instrumentista, ya que de ella depende su interpretación musical. La 
adopción de posturas forzadas y la realización de repeticiones constantes de movimientos provocan que aparezcan dichas lesiones musculares, siendo la más común la tendinitis. En el caso de los clarinetistas, hace referencia al dolor que se produce en el pulgar de la mano derecha por ser ahí donde se soporta el peso del instrumento, pudiéndose producir en la zona tendinitis o sobre uso muscular.

En relación al ámbito de nuestro estudio, THRASHER y CHESKY (1998) llevaron a cabo un estudio en intérpretes de clarinete. Participaron en dicho estudio un total de 324 clarinetistas y la edad media de los participantes fue de 34,8 años. Según dicho estudio, las zonas más propensas a sufrir patologías son las extremidades superiores (manos, muñeca, hombros, antebrazo), así como la zona de la cara donde se realiza la embocadura. De forma coincidente a las conclusiones del estudio anterior, señalaron que una de las mayores causas de las patologías más sufridas por los clarinetistas (tendinitis de Quervain) es la necesidad de soportar todo el peso del instrumento en el dedo pulgar de la mano derecha, así como el sobreuso muscular como una de las causas más frecuentes, sobre todo en mano derecha, muñeca, antebrazo y hombro.

\section{DISEÑO DE LA INVESTIGACIÓN}

A partir de estas referencias previas, el estudio que se describe en el presente artículo se planteó los siguientes objetivos de investigación:

- Conocer las patologías más comunes que sufren los clarinetistas.

- Determinar las posibles causas que inciden en la aparición de estas patologías.

- Indagar sobre los hábitos preventivos que adoptan para evitar la aparición de estas patologías

- Establecer la existencia de diferencias en función del género

El estudio que aquí se presenta ha sido realizado utilizando una metodología de investigación selectiva, cuyas características (elicitación de la respuesta, utilización de instrumentos elaborados ad hoc para la situación concreta de estudio sin responder a criterios de estandarización, selección de las variables -ítems a responder- y de carácter nomotético) es adecuada a los objetivos del estudio. Asimismo es recomendable en aquellos casos en que la aleatorización es impracticable (ANguera, 2003). Para la realización de esta investigación se ha utilizado como única variable independiente no experimental el género y como variables dependientes las respuestas al cuestionario (patologías padecidas, las causas que las provocan, etc.) medidas a través de las respuestas a los ítems del cuestionario diseñado ad hoc.

El total de participantes en el estudio fue de 41, siendo finalmente válidos solamente 38 ya que 3 de los encuestados no cumplían ninguno de los requisitos indicados con anterioridad. La media de edad de los clarinetistas participantes fue de 27,95 ( $\mathrm{SD}=6,251)$, siendo, por géneros, el $63,2 \%$ de los participantes hombres y el 36,8\% mujeres. Como criterios para la selección y participación en esta investigación se establecieron los siguientes requisitos: estar estudiando en un conservatorio superior español la especialidad de clarinete, o haber obtenido el título superior de música en la especialidad de clarinete. La elección de estos dos grupos está motivada por el incremento de las horas de estudio y tiempo dedicado al instrumento ya que existe un mayor riesgo de padecer patologías derivadas por la práctica del clarinete o se pueden hacer más evidentes en este momento de la vida musical del clarinetista.

El instrumento utilizado para obtener la información fue un cuestionario de 18 preguntas clasificadas en tres bloques diferenciados. En el cuestionario encontramos varios tipos de preguntas: cerradas, donde el encuestado debía marcar sólo una de las respuestas propuestas, como en las preguntas 2, 7 y 12 y abiertas, donde existen un conjunto de posibles respuestas donde pueden ser elegidas más de una de ellas, y donde además el encuestado puede añadir otra opción no mencionada, como por ejemplo en la 4 y 11. Existen otras que se encuentran en abanico, donde en 
virtud de si es afirmativa o negativa la primera respuesta, se pueden seguir contestando las siguientes como la 7, 8, 13 y 14 (Anexo 1).

En el primero de los bloques de preguntas de la encuesta se obtiene datos de carácter sociodemográfico (edad, género, ocupación actual y lugar donde trabajan o estudian) y sobre la experiencia profesional (años de práctica con el instrumento) y las horas semanales dedicadas al estudio del instrumento. El segundo de estos bloques enfoca las patologías padecidas por tocar el clarinete y sus posibles causas. En este apartado se obtiene información sobre cuál ha sido la primera patología que se ha producido y edad aproximada de aparición. Después de esta detección de los posibles problemas, se indaga si las molestias que las patologías producen perjudicaron a la técnica musical durante su persistencia y si tuvieron que dejar temporalmente de tocar para su total curación y durante cuánto tiempo. La última pregunta se requiere información sobre si una vez curada la patología volvió a manifestarse o simplemente se producían molestias en la misma zona afectada. Para aquellos participantes que deseasen aportar información adicional o información relevante que no se reflejara en la encuesta, se incluyó un apartado de observaciones donde podían añadir cualquier aclaración que quisiesen realizar o cualquier cuestión relacionada con la encuesta.

El estudio se llevó a cabo en tres fases. En la fase I se elaboró el cuestionario. Siguiendo los pasos descritos en la literatura al utilizar la metodología selectiva, una vez planificado el estudio se procedió a la elaboración del instrumento. Para ello, el primer paso a seguir fue la consulta de diferentes cuestionarios pertenecientes a diversos artículos de investigación relacionados con el trabajo en cuestión. A continuación se realizó una selección de las diferentes preguntas que iban a formar parte del cuestionario, dividiéndolo en tres bloques, como se ha explicado en el apartado anterior. Una vez diseñado el cuestionario se sometió a la revisión de varios expertos (profesores del ámbito universitario expertos en el diseño de este tipo de instrumentos), enriqueciendo el cuestionario con las diferentes observaciones y mejoras sugeridas. El instrumento finalmente elaborado responde al tipo de cuestionario explicativo (HERRERA y GómEZ-AMOR, 1996). El cuestionario completo y revisado se presenta en el Anexo 1. En la fase II se recogieron los datos. En primer lugar se contactó con intérpretes del clarinete que cumpliesen con los requisitos para comentarles la realización de dicho cuestionario. Una vez obtenida su conformidad, se enviaron los cuestionarios a través de e-mail. Recibidos la totalidad de los diferentes cuestionarios se realizó el vaciado de los datos en una hoja Excel. En la fase III, por último, se realizó un análisis estadístico mediante el paquete estadístico SPSS v15.

\section{RESULTADOS}

Respecto a los datos relativos a años de estudio y ocupación actual de los participantes, la media de años de práctica clarinetística es de 18,55 (rango 11-40 años, $S D=5,908$ ). En cuanto a las ocupaciones, el mayor porcentaje es estar ejerciendo como profesor $(37,2 \%)$, seguida de aquellos que son estudiantes del superior de música $(23,3 \%)$ o músicos militares $(18,6 \%)$.

La media de horas de estudio semanales del instrumento por parte de los encuestados es de 16,58 (rango 0-35, $S D=8,654$ ). Resaltar que el espectro de respuestas es muy amplio con algunos de los encuestados que afirman no estudiar ni una hora, pudiendo ser la causa el no tener tiempo para realizar esta práctica por motivos personales o profesionales. En el extremo contrario podemos encontrar participantes que estudian una media semanal de 35 horas, pudiendo ser la causa el estudio de la carrera o máster, donde el aumento de horas de estudio es considerable así como la preparación de pruebas, exámenes o conciertos.

Con lo que respecta al segundo de los bloques, las patologías sufridas, es muy destacable que de los 38 cuestionarios válidos, el 86,8\% (33 participantes) de los clarinetistas ha padecido a lo largo de su vida musical una o varias dolencias derivadas de la práctica de dicho instrumento, mientras que solamente el 13,2\% (5 participantes) manifiesta no haber padecido ningún tipo de dolencia a lo largo de su vida clarinetística. 
Entre las patologías que más han sufrido los clarinetistas participantes se encuentran el dolor de espalda y la tendinitis en la mano derecha, refiriendo en menor frecuencia otras de las patologías registradas en estudios anteriores como son el dolor en la muñeca o en el antebrazo así como problemas en la parte bucofaríngea, entre otras. Destacar que varios de ellos han sufrido más de una de estas dolencias (Tabla 1).

\begin{tabular}{|l|r|r|r|}
\hline \multicolumn{1}{|c|}{ Patologías } & \multicolumn{2}{c|}{ Respuestas } & Porcentaje \\
\cline { 2 - 3 } & \multicolumn{1}{|c|}{$\mathrm{N}^{\mathrm{o}}$} & Porcentaje & de casos \\
\hline Tendinitis en la mano & 12 & $21,1 \%$ & $36,4 \%$ \\
Dolor en la muñeca & 7 & $12,2 \%$ & $21,2 \%$ \\
Dolor en el antebrazo & 8 & $14,0 \%$ & $24,2 \%$ \\
Dolor en la espalda & 15 & $26,2 \%$ & $45,5 \%$ \\
Dolor en el cuello & 6 & $10,5 \%$ & $18,2 \%$ \\
Rinolalia & 1 & $1,8 \%$ & $3,0 \%$ \\
Faringitis & 1 & $1,8 \%$ & $3,0 \%$ \\
Tendinitis en hombro derecho & 2 & $3,5 \%$ & $6,1 \%$ \\
Dolor en el labio superior & 3 & $5,3 \%$ & $9,1 \%$ \\
Fisura en los labios y aftas bucales & 1 & $1,8 \%$ & $3,0 \%$ \\
Problemas auditivos & 1 & $1,8 \%$ & $3,0 \%$ \\
\hline Total & 57 & $100,0 \%$ & $172,7 \%$ \\
\hline
\end{tabular}

Tabla 1: Patologías sufridas por los participantes

De las primeras dolencias que aparecen por la práctica del clarinete encontramos que la patología más frecuente es el dolor de espalda con un $23,7 \%$, seguido de la tendinitis en la mano derecha, con un 18,4\%, y en menor medida otras patologías como la tendinitis de Quervein, dolor en el antebrazo y en la muñeca (Gráfico 1).

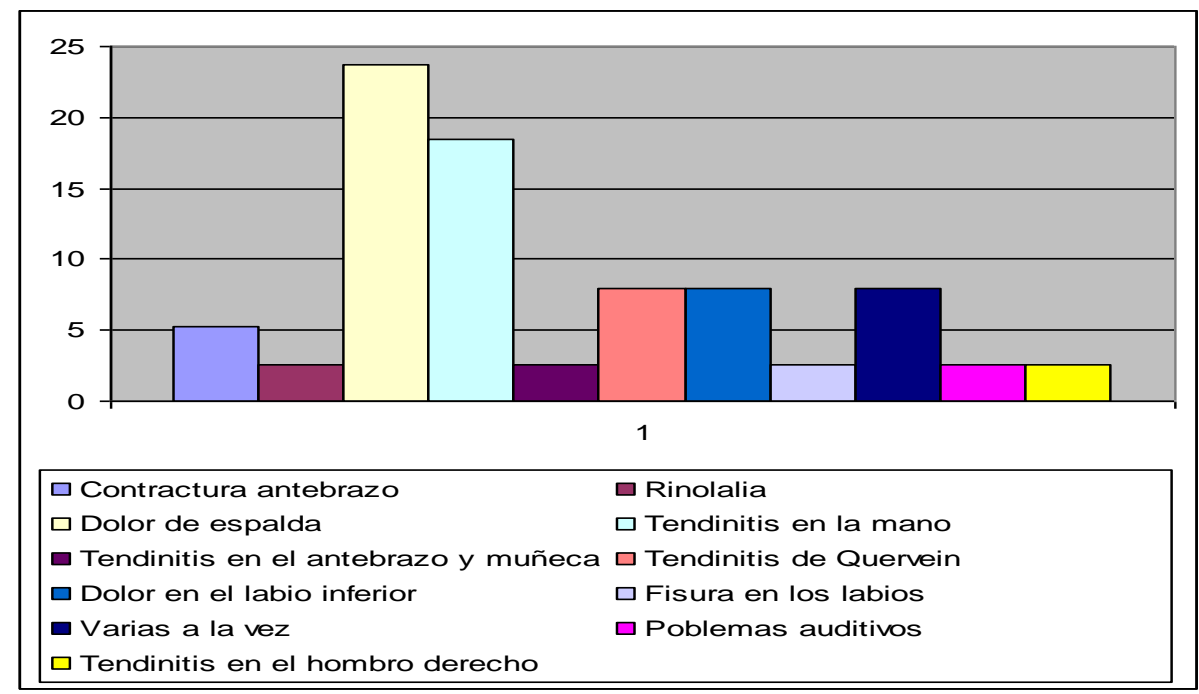

Gráfico 1: Primera patología de los clarinetistas

Según los encuestados, varias son las posibles causas de la aparición de dichas patologías, informando que mayoritariamente están relacionadas con una mala postura corporal en el momento de tocar, un aumento brusco del tiempo de estudio, así como el esfuerzo de soportar el peso del instrumento (Tabla 2). 


\begin{tabular}{|c|c|c|c|}
\hline \multirow{2}{*}{ Causa } & \multicolumn{2}{|c|}{ Respuestas } & \multirow{2}{*}{$\begin{array}{c}\text { Porcentaje } \\
\text { de casos }\end{array}$} \\
\hline & $\mathrm{N}^{\mathrm{o}}$ & Porcentaje & \\
\hline Mala posición corporal en el momento de tocar & 18 & $34,0 \%$ & $51,4 \%$ \\
\hline Aumento brusco del tiempo de estudio & 14 & $26,4 \%$ & $40,0 \%$ \\
\hline Peso del propio instrumento & 11 & $20,8 \%$ & $31,4 \%$ \\
\hline Insuficiencia velar & 2 & $3,8 \%$ & $5,7 \%$ \\
\hline Falta de estiramientos y pocas pausas de descanso & 2 & $3,8 \%$ & $5,7 \%$ \\
\hline Alergias & 1 & $1,9 \%$ & $2,9 \%$ \\
\hline Exceso de tensión & 1 & $1,9 \%$ & $2,9 \%$ \\
\hline Peso del estuche & 1 & $1,9 \%$ & $2,9 \%$ \\
\hline Mala técnica & 1 & $1,9 \%$ & $2,9 \%$ \\
\hline Presión realizada (Boca) & 1 & $1,9 \%$ & $2,9 \%$ \\
\hline Presión en la médula & 1 & $1,9 \%$ & $2,9 \%$ \\
\hline Total & 53 & $100,0 \%$ & $151,4 \%$ \\
\hline
\end{tabular}

Tabla 2: Posibles causas de la aparición de patologías en clarinetistas

Respecto a la aparición de la primera dolencia que padecen (no todos la misma), la media de edad es de 19,56 $(S D=6,026)$, edad que coincide aproximadamente con el primer curso del grado superior de música, que implica habitualmente un aumento en la exigencia y números de horas de práctica respecto a periodos de aprendizaje anteriores, situando de forma mayoritaria el rango de edad entre los 19 y 22 años, coincidiendo aproximadamente con los 4 años de los estudios superiores y por tanto con un incremento de horas de estudio y un cambio a un repertorio musical más complejo.

Respecto a las posibles causas de la dolencia de espalda que sufren los clarinetistas, la causa más importante según los encuestados, la posición incorrecta que se adopta en el momento de tocar es señalada como la más relevante a lo largo de su vida clarinetística, seguida de tensiones externas como conciertos, audiciones o miedo escénico, entre otras (Tabla 3). Al tratarse de una pregunta abierta, algunos de los encuestados han anotado varias causas para dicha dolencia.

\begin{tabular}{|l|r|r|r|}
\hline \multirow{2}{*}{ Espalda } & \multicolumn{2}{|c|}{ Respuestas } & Porcentaje \\
\cline { 2 - 3 } & \multicolumn{1}{|c|}{$\mathrm{N}^{\mathrm{o}}$} & Porcentaje & \multicolumn{1}{c|}{ de casos } \\
\hline Transporte del instrumento & 8 & $14,3 \%$ & $22,2 \%$ \\
Posición incorrecta al tocar & 27 & $48,2 \%$ & $75,0 \%$ \\
Tensiones externas & 13 & $23,2 \%$ & $36,1 \%$ \\
Misma posición tocando durante & 2 & $3,6 \%$ & $5,6 \%$ \\
mucho tiempo & 1 & $1,8 \%$ & $2,8 \%$ \\
Necesidad de ejercicio físico & 2 & $3,6 \%$ & $5,6 \%$ \\
Muchas horas tocando & 1 & $1,8 \%$ & $2,8 \%$ \\
Sujeción prolongada del instrumento & 2 & $3,6 \%$ & $5,6 \%$ \\
Exceso de tensión & 56 & $100,0 \%$ & $155,6 \%$ \\
\hline Total & &
\end{tabular}

Tabla 3: Posibles causas de la dolencia de espalda

En aquellos instrumentistas que padecieron algún tipo de dolencia, el 60,6\% de ellos manifiestan que ello provoco un empeoramiento de la técnica clarinetística, mientras que un 39,4\% indican que no vieron afectada su técnica mientras sufrían dicha dolencia. Este hecho pone de relieve la relevancia que para el desempeño de estos profesionales supone la aparición de estas patologías y, por tanto, la necesaria adopción de hábitos y actitudes que prevengan y eviten su aparición.

En cuanto a si a raíz de la dolencia o dolencias habían debido dejar de tocar momentáneamente hasta su curación, el $42,4 \%$ de los encuestados que han sufrido algún tipo de dolencia han contestado que sí, frente al 57,6\% que ha manifestado que no han tenido que dejar de tocar el clarinete por dicha patología. Entre aquellos clarinetistas participantes que han tenido que dejar de tocar, la media de tiempo que lo han tenido que hacer es de 20,29 días $(S D=26,339)$. 
De aquellas personas encuestadas que han sufrido alguna patología y después de su curación han vuelto a tener una actividad clarinetística normal, el 35\% ha notado de nuevo dolor en la misma zona afectada, el 5\% no han vuelto a tener dolor y el 60\% sólo han notado algunas molestias en la misma zona cuando se han dispuesto a tocar.

En cuanto a los hábitos preventivos, relacionados con la realización de actividad física, únicamente el $10,5 \%$ de los participantes realizan siempre estiramientos antes y después de tocar, el $44,7 \%$ en ocasiones y el $44,7 \%$ nunca. Aunque es muy bajo el porcentaje de los que realizan siempre estiramientos, el $65,8 \%$ de los encuestados muestran su acuerdo con que es importante su realización antes y después de tocar como un hábito preventivo, el $15,8 \%$ no lo considera importante y el 18,4\% lo ve importante dependiendo del tipo de estiramiento que se realice.

Respecto a la realización de actividad física, el 44,7\% la realiza varias veces por semana, el $7,9 \%$ lo practica una vez por semana, el $44,7 \%$ ocasionalmente y únicamente el $2,6 \%$ no realiza nunca actividad física. Las actividades físico-deportivas que más practican los encuestados se recogen en la Tabla 4, teniendo en cuenta que varios de ellos no realizan una única disciplina, sino varias:

\begin{tabular}{|l|r|r|r|}
\hline \multirow{2}{*}{ Deporte } & \multicolumn{2}{|c|}{ Respuestas } & Porcentaje \\
\cline { 2 - 3 } & $\mathrm{N}^{\mathrm{o}}$ & Porcentaje & \multicolumn{1}{c|}{ de casos } \\
\hline Correr & 12 & $20,7 \%$ & $32,4 \%$ \\
Senderismo & 11 & $19,0 \%$ & $29,7 \%$ \\
Natación & 12 & $20,7 \%$ & $32,4 \%$ \\
Bicicleta & 11 & $19,0 \%$ & $29,7 \%$ \\
Gimnasio & 9 & $15,5 \%$ & $24,3 \%$ \\
Baile & 2 & $3,4 \%$ & $5,4 \%$ \\
Tenis & 1 & $1,7 \%$ & $2,7 \%$ \\
\hline Total & 58 & $100,0 \%$ & $156,8 \%$ \\
\hline
\end{tabular}

Tabla 4: Actividades físico-deportivas practicadas por los participantes

Como se observa en la Tabla 4, las actividades que mayoritariamente se practican tienen un carácter de mejora cardiovascular y respiratorio, incidiendo fundamentalmente con la mejora de la resistencia aeróbica y la adopción de un estilo de vida saludable.

Por último, las respuestas a la última pregunta relativa a la incorporación por parte del profesorado de hábitos preventivos y posturales en el momento de tocar en clase el clarinete, de forma unánime el $100 \%$ de los encuestados muestran su conformidad a la adopción de dicha medida.

Al comparar los resultados en función del género, en relación a las horas de estudios los datos señalan que las mujeres dedican algo menos de tiempo al estudio semanal del clarinete, aunque destacar que mientras el 30\% de ellos estudia una media de 20 horas semanales, un 14,3\% de ellas estudian una media de 35 horas. Los datos indican que el porcentaje de mujeres participantes que han padecido alguna patología (92,9\%), ligeramente superior al porcentaje de hombres $(83,3 \%)$, confirmando ambos datos la importancia de este problema en esta especialidad instrumental en música. Por género, las patologías que más sufren los clarinetistas participantes son las indicadas en el Gráfico 2. 


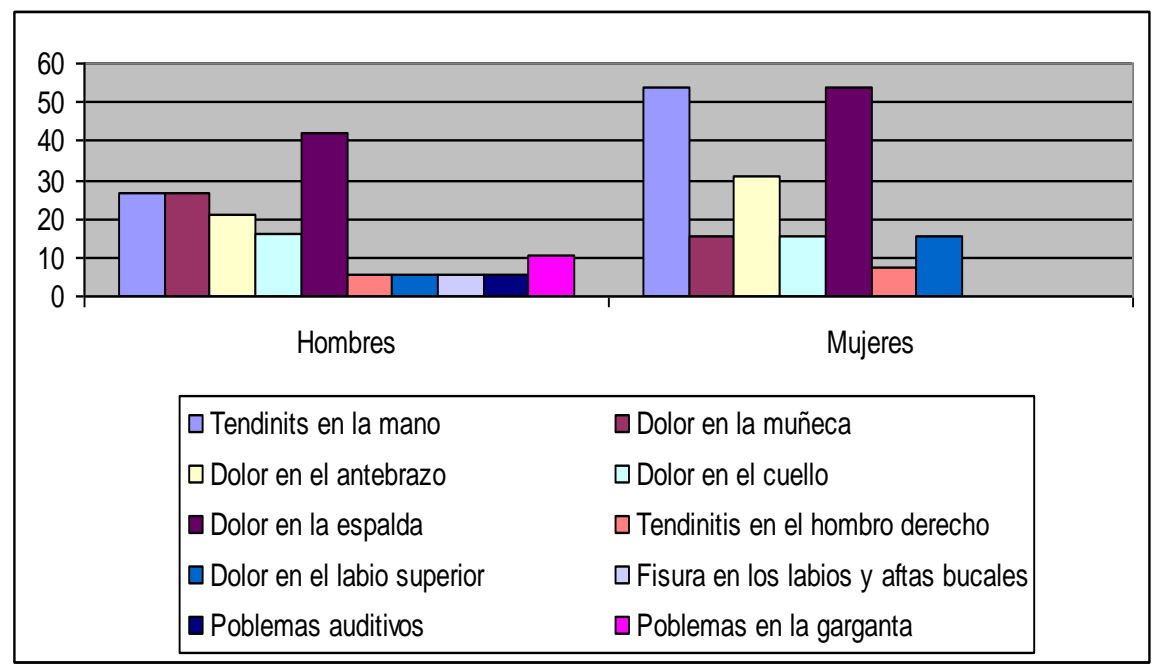

Gráfico 2: Patologías padecidas por los participantes en función del género

Estos datos revelan que entre las mujeres la tendinitis en la mano derecha y el dolor de espalda son las más comunes y, en menor medida, el dolor en el antebrazo derecho. Respecto a los hombres la variabilidad de dolencias es mayor pero en un menor grado de aparición. Destacables, al igual que en las mujeres, el dolor en el antebrazo derecho y en la espalda, refiriendo en menor medida otras como la tendinitis en la mano y el dolor en la muñeca e incorporando otras como problemas bucofaríngeos y auditivos. En cuanto a las principales causas que señalan como responsables de la aparición de patologías, las mujeres señalan en un mayor porcentaje que los hombres el aumento brusco del tiempo de estudio, como se puede comprobar en el gráfico 3.

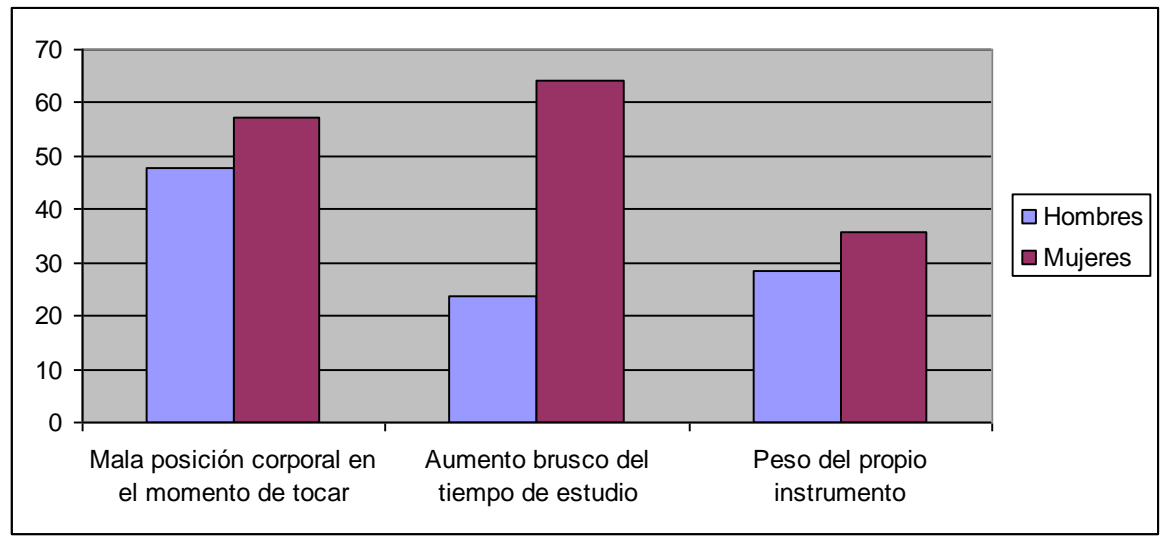

Gráfico 3: Principales causas de las patologías en función del género

La aparición de la primera dolencia es más temprana en algunas de las mujeres participantes respecto a los hombres (entre los 14 y 16 años) mientras que los hombres indican una edad posterior para dicha aparición (el 29,4\% señala los 18 años), siendo el rango 19-22 años, coincidente con la edad en que de forma mayoritaria se cursan los 4 años de los estudios superiores y, por tanto, donde se produce un incremento de horas de estudio y un cambio a un repertorio musical más complejo.

En cuanto a las posibles causas del dolor de espalda producida por la práctica clarinetística, ambos grupos coinciden, achacando esta dolencia sobre todo a la posición incorrecta que se adopta al tocar (Gráfico 4). 


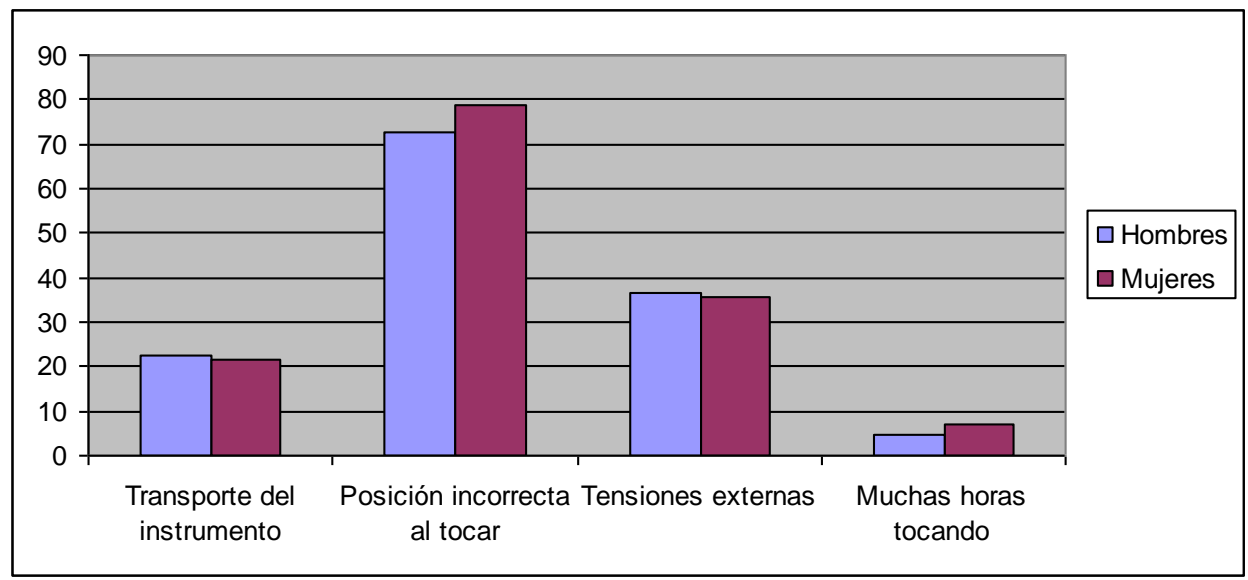

Gráfico 4: Posibles causas del dolor de espalda en función del género

Es importante destacar que la aparición de las patologías provocó un empeoramiento de la técnica instrumental (un 71,4\%) a las clarinetistas participantes, frente a un porcentaje menor de hombres que se vio afectado con este empeoramiento (55\%). Con respecto a tener que dejar de tocar el clarinete durante el periodo de curación de las dolencias padecidas, los valores entre géneros son muy similares, señalando que sí el $40 \%$ de los hombres y el $46,2 \%$ de las mujeres respectivamente, lo que supone, aproximadamente, la mitad de los participantes.

Respecto a volver a manifestarse la patología en la misma zona una vez que vuelven a su vida clarinetística, es relevante (aunque no se han hallado diferencias estadísticamente significativas quizás debido al pequeño tamaño de la muestra) la diferencia existente entre géneros. Entre los hombres siguen teniendo dolor un $41,7 \%$, solamente sienten molestias el $50 \%$ y únicamente el $8,3 \%$ informa no presentar ningún síntoma al respecto. Entre las mujeres el dolor disminuye respecto a los hombres (un 25\%), pero el porcentaje de las que manifiestan seguir padeciendo algunas molestias es bastante superior respecto a ellos (un 75\%) (Gráfico 5).

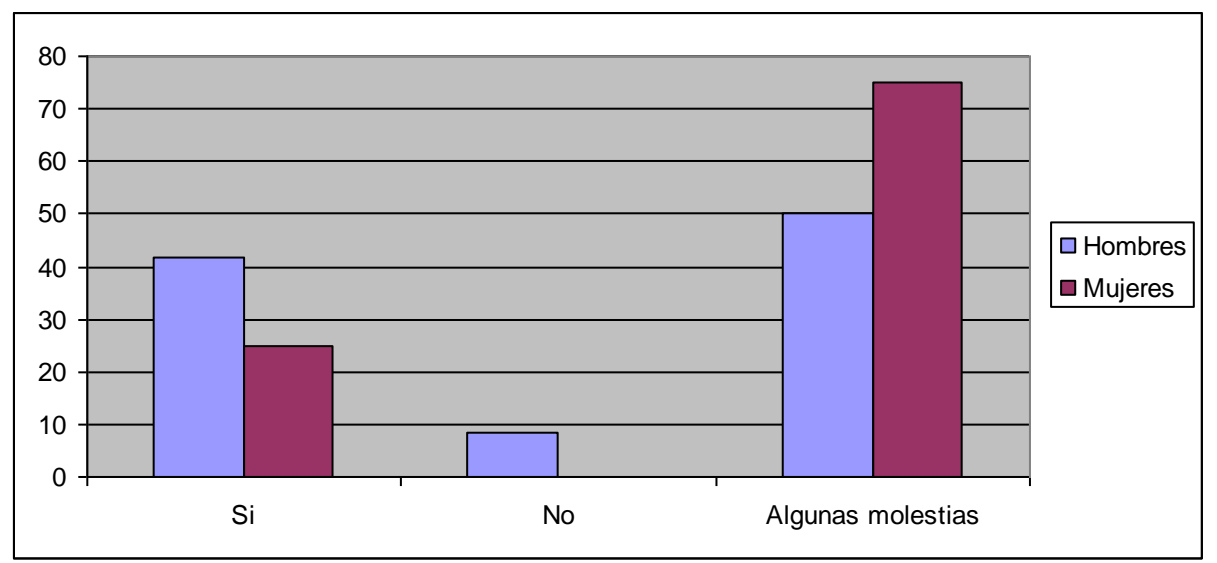

Gráfico 5: Reaparición del dolor en la misma zona en función del género

Respecto a la adopción de hábitos preventivos, el porcentaje de mujeres que realizan estiramientos antes y después de tocar es mayor que el de los hombres (Gráfico 6), siendo el 78,6\% las que sí lo ven importante frente a un $60,9 \%$ de los hombres. 


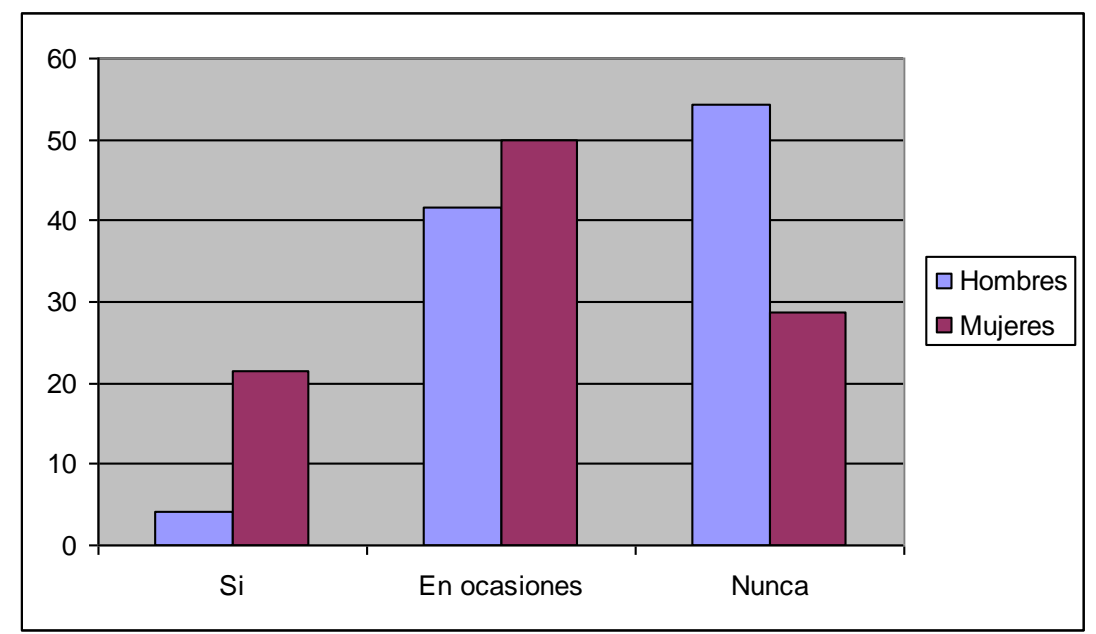

Gráfico 6: Realización de ejercicios de estiramiento, antes y después de tocar, por géneros

Referente a la práctica de actividad física, el porcentaje es similar entre ambos géneros, siendo las mujeres las que presentan un mayor porcentaje de práctica varias veces por semana respecto a los hombres (Gráfico 7).

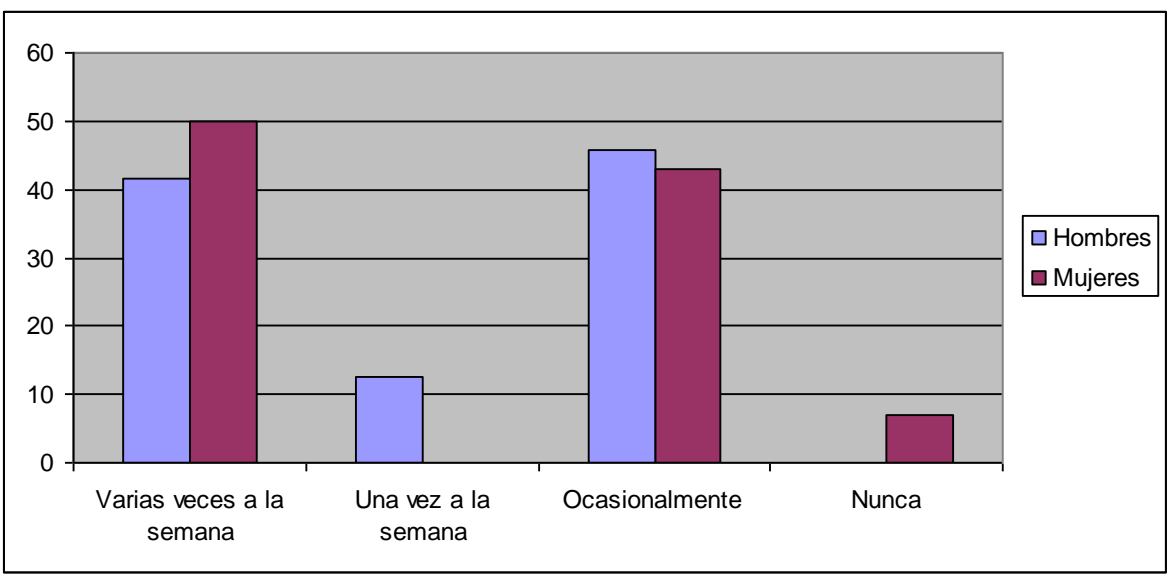

Gráfico 7: Práctica de actividad física en función del género

Respecto a las diferentes actividades físicas que realizan, los hombres practican más correr y bicicleta, mientras que las mujeres practican más natación, senderismo e ir al gimnasio (Gráfico 8).

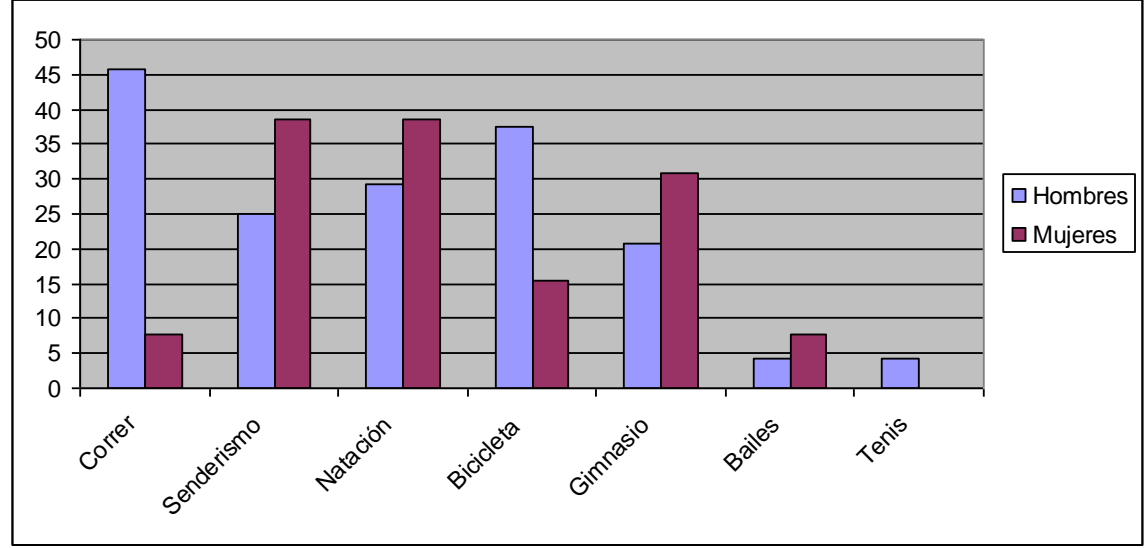

Gráfico 8: Tipo de actividad física practicada en función del género

\begin{abstract}
ANÁLISIS
El presente estudio tenía como objetivos conocer las patologías más comunes que sufren los clarinetistas, determinar las posibles causas que pueden afectar a la aparición de patologías, indagar sobre los hábitos preventivos que adoptan para evitar la aparición de estas patologías y establecer la
\end{abstract}


existencia de diferencias en función del género. Los datos encontrados en el presente estudio confirman que un gran número de participantes, a lo largo de su vida como clarinetista, ha sufrido algún tipo de patología derivada de esta práctica musical (un 86,8\%), siendo coincidente este dato con los encontrados en otros estudios realizados como los de ROSSET, ROSINÉS y SALÓ (2000) con un 77,9\% de afectados, de FRANK y VON MÜHLEN (2007) con un 70\% de afectados y de JOUBREL y sus colaboradores (2001) con un 76,6\% de participantes con dolencias, pudiendo ser el motivo de un porcentaje inferior en estos estudios el hecho de que se dirigieron a conocer esta cuestión en varios instrumentos diferentes y tal vez, dependiendo del instrumento, se pueden padecer más o menos patologías. Estos datos también son similares a los señalados por NAVIA y sus colaboradores (2007) en un estudio con participantes que eran miembros de orquestas profesionales (un 68,7\% de ellos habían padecido dolencias). Este elevado porcentaje pone en evidencia que no se está realizando una óptima enseñanza del clarinete con respecto a las posiciones más correctas que se deben adoptar en el momento de tocar, saliendo a la luz problemas cuando el propio clarinetista incrementa sus horas de estudio, comenzando a notar molestias que a posteriori se pueden convertir en dolor, interfiriendo así en su avance como intérprete.

Entre las patologías más comunes sufridas por los clarinetistas participantes encontramos el dolor de espalda (26,3\%), tendinitis en la mano (21,1\%), dolor en el antebrazo (14\%) y dolor en la muñeca $(12,3 \%)$ siendo de menor incidencia otras ya indicadas en el apartado de los resultados. En los diferentes estudios consultados se constata coincidencias con algunos de los resultados obtenidos en el presente estudio. Así, Rosset, Rosinés y SALÓ (2000) señalan que las patologías más comunes de los instrumentistas son, en general, aquellas que suceden en la parte musculoesquelética, en particular en las extremidades superiores, zona cervical y la zona dorsal de la espalda. En concreto en los instrumentos de viento con lengüeta simple, al que pertenece el clarinete, encuentran diferencias, ya que el dolor cervical es el más elevado, siendo relacionado en muchas ocasiones con dolor de espalda en la parte superior, coincidiendo con los datos encontrados en el actual trabajo de investigación.

En el estudio de FRANK y VON MÜHLEN (2007), se hace referencia como patologías a diferentes tendinitis en las extremidades superiores y el sobreuso muscular como las más comunes, la primera de ellas afectando también en gran medida a los clarinetistas participantes del presente estudio. En otro de los estudios, JOUBREL y sus colaboradores (2001) señalan tres patologías como las más significativas de su trabajo, como son el sobreuso muscular, el síndrome del túnel carpiano y la distonía focal, ésta última relacionada con un trastorno neurológico, pero remarcan que el 60,9\% de los encuestados sufren dolores en la parte de la columna, sobre todo en la parte cervical y dorsal y el $52,5 \%$ en las extremidades superiores, en concreto en las manos y en brazos, coincidiendo plenamente con las zonas de las patologías más destacadas entre los clarinetistas participantes en el presente estudio. THRASHER y CHESKY (1998), con su estudio específico a clarinetistas, vuelven a remarcar el hallazgo anterior respecto a las zonas más perjudicadas en las extremidades superiores, pero no indican nada respecto a la musculatura de la espalda, ratificando que es más afectado el pulgar de la mano derecha, dicha mano y todo el brazo derecho, en general, que el izquierdo como consecuencia de la interpretación musical con el clarinete. Es evidente que estas patologías suceden en su mayoría en las extremidades superiores y en la zona superior de la espalda, como se indican también en los anteriores estudios consultados, coincidiendo con las que el clarinetista tensa más en el momento de tocar, tanto en el momento de estudio, de las audiciones y de los conciertos, provocando a la larga que esas tensiones generen problemas que perjudicarán negativamente al clarinetista.

Respecto a los valores encontrados en función del género, parecen indicar que existen ciertos factores que favorezcan o faciliten la aparición de las patologías dependiendo de si se es eres hombre o mujer, encontrando en nuestro estudio que un porcentaje mayor de mujeres habían sufrido patologías $(93 \%)$ respecto a los hombres $(83,3 \%)$. Estos mismos datos coinciden con los reseñados en estudios previos, siendo más elevado el porcentaje de mujeres que padecen patologías entre todos los instrumentistas (FRANK y VON MÜHLEN, 2007), apuntando que la posible causa de estos 
resultados sea la constitución física de la mujer, con una menor fuerza muscular y, en ocasiones, con manos más pequeñas respecto a ellos. JOUBREL y sus colaboradores (2001) también coinciden en que las mujeres son más propensas a padecer patologías y, en concreto, aquellas patologías que están relacionadas con el síndrome del sobreuso muscular. GREER y PANUSH (1994) señalaron la genética de la mujer como posible causa de estos resultados. Los anteriores estudios están relacionados con diferentes instrumentos pero el estudio de THRASHER y CHESKY (1998), únicamente con clarinetistas, también presenta coincidencia con dichos datos, siendo las mujeres las que más sufren patologías, corroborando los datos obtenidos en el presente estudio. Existen varias cuestiones en el estudio actual que deben ser mencionadas como la aparición más temprana en las mujeres de las primeras dolencias, aproximadamente entre los 14 y 16 años, con respecto a los hombres. Esto puede relacionarse con la constitución física de la mujer y el hecho de que un alto porcentaje de ellas sufran patologías a lo largo de su vida clarinetística.

Es destacable también el dato referente al empeoramiento de la técnica como consecuencia de la aparición de la patología, ya que de los participantes en el actual estudio sí que se vieron afectados por ello (un 60,6\%), pero es más sorprendente los resultados obtenidos si analizamos estos datos por géneros, ya que el $71,4 \%$ de las mujeres han notado este empeoramiento respecto al $55 \%$ de los hombres; esto puede deberse nuevamente a la constitución física de la mujer, que se ve más afectada por dichas patologías. Estos datos muestran diferencias relevantes en comparación con otros estudios, como el de NAVIA y sus colaboradores (2007), donde el 100\% de los participantes vieron negativamente afectada su técnica por las patologías padecidas.

Al igual que con las actividades físicas, la musculatura del hombre y la mujer son diferentes, esto sucede también con los instrumentistas, ya que aunque no sea evidente visualmente el esfuerzo físico que se realiza mientras se toca, si se puede comprobar como el clarinetista después de realizar un concierto está cansado físicamente por el esfuerzo que realiza en él. Si las mujeres muscularmente son más sensibles que los hombres, todas las afirmaciones anteriores se ratifican, ya que si no se está bien preparada físicamente, los problemas musculares pueden empezar a suceder en cualquier momento siendo perjudicial para la clarinetista, incluyendo en muchas ocasiones periodos de descanso para la curación total o parcial de la patología padecida, siendo un tiempo en el cual se deja de tocar y por tanto paraliza por completo la actividad interpretativa del músico.

Respecto a las causas de dichas patologías, podemos destacar tres de ellas como las consideradas más importantes por los clarinetistas participantes: en orden de importancia, mala posición corporal en el momento de tocar (34\%), aumento brusco en el tiempo de estudio $(26,4 \%)$ y el peso del propio instrumento $(20,8 \%)$. Otros estudios consultados corroboran dichos resultados como el llevado a cabo por ROSSET, RosinéS y SALÓ (2000), donde señala la segunda de estas causas un $43,8 \%$ de los encuestados, siendo también informada por los participantes en el estudio de GREER y PANUSH (1994), acompañando al aumento de las horas de estudio un incremento en la dificultad de las obras a interpretar. FRANK y VON MÜHLEN (2007) coinciden en el aumento del tiempo de estudio del instrumento, resaltando otras causas no señaladas en el presente estudio como la técnica utilizada en el instrumento, ya que puede ser deficiente y por ello producir patologías, mientras que DOMMERHOLT (2010) encontró que los participantes en su estudio señalaban las posturas forzadas, pudiendo entenderse ésta como similar a la primera de las que señalan los participantes en este estudio. Este mismo autor indica asimismo como causa las repeticiones constantes de movimiento durante el tiempo de práctica, también señalada por GREER y PANUSH (1994), y especifica como causa del dolor en el pulgar de la mano derecha, en concreto en el clarinete, el soporte del peso de dicho instrumento, cuestión coincidente con la tercera de las causas del presente estudio. THRASHER y CHESKY (1998), respecto a las cusas de las patologías específicas en clarinetistas, también mencionan el peso del instrumento como causa de muchas de las que se producen en las extremidades superiores, en especial, en la mano y el pulgar derecho.

Es evidente que las dos primeras causas de las patologías que apuntan los participantes de dicho estudio, mala posición corporal en el momento de tocar (34\%) y aumento brusco en el tiempo de estudio $(26,4 \%)$ están motivadas por una mala enseñanza del clarinetista desde sus inicios, ya que el 
profesorado, en muchas de las ocasiones, únicamente atiende a si el alumno realiza bien el estudio o la obra, si realiza los matices, las articulaciones o la musicalidad, dejando en un segundo plano la posición corporal que adopta el alumnado en el momento de tocar y el calentamiento previo antes de abordar el grueso de la clase. Si desde que el alumnado empieza con el instrumento se tuviese en cuenta estos últimos aspectos, y el clarinetista fuera consciente desde el principio de todo lo que puede suceder si no se realiza una buena posición corporal y calentamientos previo a la interpretación, es muy probable que descendería notablemente las patologías y por ello el gran porcentaje de ellos que las sufren a lo largo de su vida como alumnos y profesionales. Es necesario una nueva concepción del profesorado respecto a la enseñanza del clarinete, incluyendo nuevos aspectos que favorezcan a largo plazo al instrumentista en todos los aspectos, no únicamente en los musicales, y todo ello empieza con una concienciación en todos los aspectos de las enseñanzas de un instrumento desde el inicio, no cuando el problema sea evidente y solucionarlo sea mucho más complicado.

En relación a los hábitos de prevención y realización de actividad física, aunque únicamente el $10,5 \%$ de los participantes realiza siempre estiramientos antes y después de tocar, el porcentaje de los que concede gran importancia a realizarlos diariamente es de un $65,8 \%$, encontrando a este respecto una cierta contradicción entre la acción y el pensamiento de los participantes. Esto puede ser debido a la forma de estudiar ya que muchos piensan que es importante y necesario pero no los realizan posiblemente por falta de hábito en el momento del estudio o los realizan únicamente cuando notan algún tipo de molestia, aunque sean conscientes de su importancia para la prevención en la aparición de patologías. NAvíA y sus colaboradores (2007) coinciden en la importancia del calentamiento antes y después de tocar, incluyendo otras acciones como la educación preventiva de las dolencias en el momento de tocar como parte importante de la enseñanza musical desde los inicios de los estudios o la importancia del estado físico del músico. A este respecto, el $100 \%$ de los participantes manifiestan su acuerdo con la importancia de incorporar hábitos preventivos y posturales en el momento de tocar en la clase de clarinete por parte del profesorado.

Respecto a la realización de actividad física por los instrumentistas, NAvía y sus colaboradores (2007) encontraron respecto a los hábitos deportivos un porcentaje mucho mayor de músicos que no realizan nada de actividad física (50\%) que el encontrado en el presente trabajo (2,6\%). Estos datos, tomándolo con la necesaria precaución dado el pequeño tamaño de la muestra de este estudio, parece indicar una mayor concienciación, en general, hacia la necesidad de mantener un nivel de actividad medio/alto en relación con la consecución de estilos de vida activos y saludables y de forma específica asumir la importancia que niveles adecuados de desempeño físico-motor tienen en la realización de su actividad como instrumentistas y en la prevención de las patologías manifestadas por ellos. Como indican POTTER y JONES (1995), es importante estar físicamente bien para cualquier músico ya que desarrolla varios aspectos importantes para la prevención de patologías gracias al fortalecimiento de los músculos y de la mejora de la resistencia durante la interpretación musical.

\section{CONCLUSIONES}

De los resultados encontrados cabe extraer las siguientes conclusiones principales:

- El 86,8\% de los clarinetistas participantes en el estudio han declarado haber padecido una o varias patologías derivadas de su práctica musical a lo largo de su vida como clarinetista.

- Las patologías más relevantes señaladas por los participantes han sido el dolor de espalda $(26,3 \%)$ y tendinitis en la mano derecha $(21,1 \%)$, siendo algunas de sus posibles causas una mala adopción corporal en el momento de tocar (34\%), un aumento brusco en el tiempo de estudio $(26,4 \%)$ y el peso del propio instrumento sobre el pulgar derecho $(20,8 \%)$. 
- Se detecta la existencia de una mínima, por no decir casi nula, prevención mediante la realización de estiramientos antes y después de tocar, ya que sólo el 10,5\% de los participantes manifiestan realizar estiramientos siempre.

- Estos datos ponen de manifiesto una clara contradicción entre pensamiento y acción, contradicción debida seguramente a una falta de hábitos bien consolidados a la hora de comenzar y finalizar la práctica de interpretación, y a una ausencia de una verdadera concienciación sobre la importancia que ello tiene en el desarrollo de la carrera del instrumentista y en los efectos positivos a la hora de alcanzar las máximas cotas de desempeño como instrumentista musical.

Estos hallazgos deberán servir en el futuro para orientar a profesores y alumnos sobre la importancia de adoptar en todo momento posiciones adecuadas del cuerpo en el momento de la interpretación a partir del conocimiento de los efectos que sobre los niveles de tensión muscular ejercen dichas posiciones respecto a los grupos musculares participantes de la acción, así como a incrementar de modo progresivo, atendiendo a un adecuado ciclo de trabajo-descanso, las horas de práctica a lo largo del proceso de aprendizaje y consolidación de la técnica instrumental.

\section{Referencias}

AlteNMÜLlER, E. (2003): "Focal dystonia: Advances in brain imaging and understanding of fine motor control in musicians". Hand Clinics, 19(3), 523-538.

AnguerA, M. T. (2003): "La metodología selectiva en la psicología del deporte". En A. HERnÁndez (coord.): Psicología del deporte (Vol. 2). Metodología (págs. 74-96). Buenos Aires, Efdeportes

BARR, A. E..; BARBE, M. F. (2002): "Pathophysiological tissue changes associated with repetitive movement: A review of the evidence". Physical Therapy, 82(2), 173-187.

BRANDFONBRENER, A. G.; RoBSON, C. (2002): "A review of 111 musicians with focal dystonia seen at a performing artist's clinic 1985-2002". Movement Disorders, 17, 1135-1138.

CANTÓ, F. J. (2008): Los brazos del clarinetista. Consultado en http://www.csicsif.es/andalucia/modules/mod ense/revista/pdf/Numero 5/FRANCISCO J CANTO CAR RILLO.pdf, el 27 de abril de 2010.

Comella, C.L.; WuU, J.; Stebbins, G.T.; ChmurA, T. (2003): "Rating scales for dystonia: A multicenter assessment". Movement Disorders, 18, 303-312.

Diethelm, U. (2010): "Síndrome de sobrecarga en las manos". Eufonía. Didáctica de la Música, 48, 23-27.

DOMMERHOLT, J. (2010): "Performing arts medicine - Instrumentalist musicians: Part II Examination”. Journal of Bodywork \& Movement Therapies, 14(1), 65-72.

ELSHENSOHN, S. (2010): Espalda y psique, todo lo que nos puede pasar por la espalda”. Eufonía. Didáctica de la Música, 48, 9-15.

Frank, A.; Von MüHLEN, C. A. (2007): “Queixas musculoesqueléticas em músicos: Prevalencia e fatores de risco". Revista Brasileira de Reumatología, 47(3), 188-196.

GREER, J. M.; PANUSH, R. S. (1994): "Musculoskeletal problems of performing artists". Baillière's Clinical Rheumatology, 8, 103-135.

HERRERA, E.; GÓMEZ-AMOR, J. (1996): "Diferencias psicofisiológicas y de personalidad entre mujeres deportistas y sedentarias”. En E. PÉrez CóRdOBA y J. C. CARACUEL (eds.): Psicología del Deporte. Investigación y aplicación (págs. 155-159). Málaga, Instituto Andaluz del Deporte de la Junta de Andalucía.

Hislop, H. J.; MONTGOMERY, J. (2002): Pruebas funcionales musculares. Madrid: Marban.

HoppmanN, R. A.; PATRON, N. A. (1989): "A review of musculoskeletal problems in instrumental musicians". Seminars in Arthritis and Rheumatism, 19(2), 117-126.

JABUSCH, H.C.; AltenMÜLleR, E. (2006): "Focal dystonia in musicians. From phenomenology to therapy". Advances in Cognitive Psychology, 2, (2-3), 207-220. 
Joubrel, I.; Robineau, S.; PÉtrilli, S.; Gallien, P. (2001): "Pathologies de l'appareil locomoteur du musicien. Etude épidémiologique". Annales de Réadaptation et de Médecine Physique, 44 (2), 72-80.

Navia, P.; ArráEZ, A.; Alvarez, P.; ArdíACA, L. (2007); "Incidencia y factores de riesgo de dolor cervical en músicos de orquestas españolas". Mapfre Medicina, 18(1), 27-35.

Orozco, L.; Cervello, J. (1996): “Alteraciones osteomusculares y de los nervios periféricos. Traumatología”. En L. OrozCo y J. SolÉ (eds.): Tecnopatías del músico (pp. 133-161). Barcelona: Aritza.

PotTer, P.; Jones, I. C. (1995): "Medical problems affecting musicians". Canadian Family Physician, 41, 2121-2128.

Rosset, J. (2003): "Profesión de riesgo". Doce Notas, 38, 33.

Rosset, J. (2004): "Problemas de salud de los músicos y su relación con la educación". Consultado en http://www.institutart.com/pdf/salud musico y educación.pdf, el 23 de enero de 2010.

Rosset, J.; Rosinés, D.; SALÓ, J. M. (2000): "Identification of risk factors for musicians in Catalonia (Spain)". Medical Problems of Performing Artists, 15, 167-174.

Rosset, J.; FÀbregues, S. (2005): A tono. Ejercicios para mejorar el rendimiento del músico. Barcelona, Paiporta.

SCHARF, S. P. (2010): “Aspectos de las enfermedades funcionales y estructurales de la espalda en los músicos”. Eufonía. Didáctica de la Música, 48, 16-22.

SCHAWARZENBACH, S. (2010): "Enseñanza del instrumento y medicina musical, más vale prevenir que curar". Eufonía. Didáctica de la música, 48, 50-59.

THRASHER, M.; CHESKY, K. S. (1998): "Medical problems of clarinettists: Results from the UNT Musicians Health Survey”. The Clarinet, 25, 24-27. 


\section{Anexo I: Cuestionario}

\section{FILIACIÓN PERSONAL}

1. Edad:

2. Género: Hombres ___ Mujer

3. ¿Cuántos años hace que tocas el clarinete?

4. Ocupación actual:

- Estudiante de superior de música

- Profesor

- Músico militar

- Músico de orquesta

- Otros (especificar)

5. Localidad y provincia de estudios (en caso de serlo) o de trabajo

6. Horas aproximadas de estudio del instrumento semanalmente

PATOLOGÍAS

7. ¿Has padecido alguna enfermedad o dolencia que estuviese relacionada con la práctica musical del clarinete?

a) Sí

b) No

8. Si es afirmativo, cuál de las siguientes (se pueden marcar varias):

a) Tendinitis en la mano

b) Dolor en la muñeca

c) Dolor en el antebrazo

d) Dolor en la espalda (especificar si en parte superior o inferior)

e) Dolor en el cuello (parte cervical)

f) Otros, especificar

9. ¿A qué crees que son debidas estas dolencias?

a) Mala posición corporal en el momento de tocar

b) Aumento brusco del tiempo de estudio

c) Peso del propio instrumento

d) Otros (especificar)

10. Edad aproximada de la aparición de la primera dolencia

- ¿Cuál fue?

11. La espalda es una de las partes que más sufren los clarinetistas y los instrumentistas en general ¿Cuál crees que es la causa del posible dolor de espalda?
a) Transporte del instrumento
b) Posición incorrecta al tocar
c) Tensiones externas
d) Otros (especificar)

12. ¿Las molestias hicieron provocar un empeoramiento de la técnica clarinetística?

a) Sí

b) No

13. A raíz de estas dolencias ¿Has tenido que dejar de tocar el clarinete durante algún tiempo para su total curación?

a) Sí ¿¿Durante cuánto tiempo?

b) No

14. Si la respuesta anterior es afirmativa, después de su curación ¿Has vuelto a tener dolor en la misma zona?

a) Sí

b) No

c) Únicamente algunas molestias 